Pecvnia, Monográfico (2008), pp. 105-128

\title{
Calidad del servicio percibida por clientes de entidades bancarias de Castilla y León y su repercusión en la satisfacción y la lealtad a la misma*
}

\author{
José-Á. Miguel-Dávila \\ jam.davila@unileon.es \\ Universidad de León
}

Organización de Empresas

Fac. de Ciencias Económicas y Empresariales

Campus de Vegazana, $\mathrm{s} / \mathrm{n}$

24071 León (España

Marcela Flórez-Romero

marcelaflorez@ufps.edu.co

Univ. Francisco de Paula Santander

Avda. Gran Colombia, 12E-96B Colsag.

San José de Cúcuta (Colombia)

El propósito de la investigación está enmarcado en el estudio de la calidad del servicio que prestan las entidades bancarias. El objetivo de la investigación ha sido el de identificar los factores que determinan la calidad del servicio que
The aim of this paper is to analyze the quality of the services offered by banking entities. The objective of the research has been to identify the key factors determining the service quality as perceived by the clients of

Los autores agradecen la financiación recibida de la Consejería de Economía y Empleo de la Junta de Castilla y León a través del proyecto titulado "Medición de la calidad del servicio en las entidades financieras de la Comunidad de Castilla y León". 
perciben los clientes de las entidades bancarias de Castilla y León, y de cómo la calidad influye en su satisfacción. Así mismo, se trata también de identificar cómo la satisfacción de los clientes por el servicio prestado afecta a su lealtad hacia la entidad.

Mediante un análisis factorial de componentes principales, se encontraron los factores que influyen en la calidad: i) aspectos físicos (estructura física, ubicación conveniente, equipamiento moderno, vigilancia, aspecto limpio, etc.); ii) aspectos de la prestación del servicio (operaciones y servicios convencionales que realiza y ofrece la entidad, confianza y conocimiento que transmite el personal, atención personalizada, tiempos de respuesta, reputación de la entidad); y iii) nuevas tecnologías (cajeros, Internet o banca telefónica). Posteriormente, los resultados del modelo de ecuaciones estructurales, muestran una gran influencia de los aspectos de la prestación del servicio en la calidad del servicio, así como la demostración de la calidad como antecedente de la satisfacción y la influencia que ejerce la calidad en la satisfacción y ésta en la lealtad del cliente a la entidad bancaria.

Palabras clave: Calidad del servicio / Satisfacción / Lealtad / Ecuaciones Estructurales / Sector bancario. banking entities in Castilla y León; it is important to note the way in which quality influences the customers' satisfaction. Others goals of the research focus on how the clients' satisfaction on the perceived service affects their loyalty to the entity.

Through a factor analysis of the main components, the key features influencing quality were found: i) physical aspects (physical structure, convenience location, modern equipment, etc.), ii) aspects of service's performance (conventional operations and services which the bank offers and performs, personalized service, reputation of the entity, etc.), and iii) new technologies (cash dispenser, internet or phone banking). Afterwards, the results of the structural equations model indicate a great influence of the aspects of service's performance in the service quality. They also demonstrate that quality appears as an antecedent of satisfaction and that there is an influence of quality in satisfaction and of satisfaction in the loyalty of the customer to the banking entity.

Key words: Service Quality / Satisfaction / Loyalty / Structural Equations / Banking Sector.

\section{INTRODUCCIÓN}

En las últimas décadas, y como consecuencia de la puesta en marcha por parte de los gobiernos de políticas sobre globalización y liberación de los mercados, los clientes son cada vez más críticos con la calidad del servicio (Gayathri et al. 2005: 123). Un camino que han decidido tomar las entidades del sector bancario para adaptarse al entorno competitivo ha sido el de reorientar su filosofía para enfocarse en el servicio al cliente, con el fin de introducir el concepto de calidad del servicio en la mente sus clientes, buscando con ello, su crecimiento (Sharma y Mehta 2004: 218).

El objetivo del presente trabajo es identificar cuáles son los factores que determinan la calidad del servicio que perciben los clientes de entidades bancarias, y cómo influye ésta en la satisfacción del cliente; 
asimismo, también se trata de identificar cómo la satisfacción del cliente afecta a la lealtad hacia la entidad.

Para el logro de este objetivo, el trabajo se estructura en dos partes. En primer lugar, se realiza una revisión de la literatura sobre las investigaciones más relevantes en calidad del servicio, determinando las aportaciones más valiosas para nuestra investigación, que nos ayudan a determinar los factores que influyen en ella: i) aspectos físicos (estructura física de la oficina, ubicación conveniente, equipamiento moderno, vigilancia, aspecto limpio, etc.); ii) aspectos de la prestación del servicio (operaciones y servicios convencionales que realiza y ofrece la entidad, confianza y conocimiento que transmite el personal, atención personalizada, tiempos de respuesta, reputación de la entidad, etc.); y iii) nuevas tecnologías (cajeros, Internet o banca telefónica).

La segunda parte del trabajo recoge la parte empírica, subdividida en diferentes partes: (i) la metodología utilizada para la construcción del instrumento de medida; (ii) validez y fiabilidad de la escala de medida; y, (iii) contraste de hipótesis mediante la modelización de ecuaciones estructurales (MEE).

HIPÓTESIS

\section{REVISIÓN DE LA LITERATURA Y PLANTEAMIENTO DE}

\subsection{Calidad del servicio en entidades bancarias}

El interés en la calidad de servicio ha crecido enormemente desde los años ochenta (Grönroos 1983; Parasuraman et al. 1985; Lewis y Klein 1987; Gummesson y Grönroos 1988; Zeithaml et al. 1988). Hoy en día, los bienes y servicios no sólo tienen que ser aptos para el uso que se les ha asignado, sino que además tienen que igualar e incluso superar las expectativas que los clientes han depositado en ellos. En la bibliografía sobre el estudio de los servicios se ha discutido una serie de peculiaridades que les diferencian de los bienes (ver Cuadro 1) y que pueden condicionar las decisiones a tomar en la gestión de las entidades bancarias para ofrecer una buena calidad en los servicios que prestan.

Un servicio bancario es la actividad que desarrollan las entidades financieras como empresas de servicios; es decir, todas aquellas acciones que se establecen para servir a los clientes (González Aponcio 2001: 96). El servicio bancario se caracteriza por sufrir rápidos cambios en 
su ambiente (Jayawardhena 2004: 186), algunos gracias a la tecnología, y que, relacionada con la información, resulta ser cada vez más importante (Shih y Fang 2006: 62). La mayoría de las investigaciones basadas en el estudio de la calidad del servicio que prestan las entidades bancarias, se han fundamentado básicamente, en definir las dimensiones de la calidad y construir un modelo para medirla, siempre con el objetivo de mejorar la calidad del servicio (González Aponcio 2001: 105).

Cuadro 1: Características diferenciadoras entre bienes y servicios

\begin{tabular}{|c|c|c|}
\hline \multirow{10}{*}{ 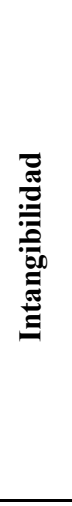 } & Servicios & Bienes \\
\hline & Son intangibles & Son tangibles \\
\hline & No se pueden almacenar & Se pueden almacenar \\
\hline & No se pueden transportar & Se pueden transportar \\
\hline & No hay transferencia de propiedad & Transferencia de propiedad por la compra \\
\hline & No se pueden patentar & Se pueden patentar \\
\hline & Difícil establecer su coste & Facilidad estableciendo el coste \\
\hline & El output es un proceso o una actividad & El output es una cosa \\
\hline & Tienen consecuencias & Tienen formas \\
\hline & No pueden medirse & Pueden medirse \\
\hline \multirow{5}{*}{ 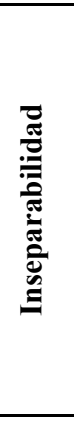 } & $\begin{array}{l}\text { No se pueden separar las funciones de } \\
\text { producción, distribución y consumo }\end{array}$ & $\begin{array}{l}\text { Las funciones de producción y } \\
\text { distribución separadas del consumo }\end{array}$ \\
\hline & $\begin{array}{l}\text { Se prestan tras una solicitud hecha por } \\
\text { el cliente }\end{array}$ & $\begin{array}{l}\text { Se pueden fabricar antes de que lo pida el } \\
\text { cliente }\end{array}$ \\
\hline & $\begin{array}{l}\text { La satisfacción del trabajador es } \\
\text { fundamental }\end{array}$ & $\begin{array}{l}\text { La satisfacción del trabajador no es } \\
\text { prioritaria }\end{array}$ \\
\hline & $\begin{array}{l}\text { Capacidad no usada es capacidad } \\
\text { perdida }\end{array}$ & $\begin{array}{l}\text { Las empresas pueden 'jugar' con la } \\
\text { capacidad }\end{array}$ \\
\hline & $\begin{array}{l}\text { El cliente es parte del proceso de } \\
\text { producción }\end{array}$ & $\begin{array}{l}\text { El cliente no participa en el proceso de } \\
\text { producción }\end{array}$ \\
\hline \multirow{3}{*}{ 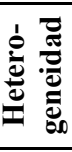 } & Son variados & Se pueden estandarizar \\
\hline & El cliente percibe riesgo elevado & El cliente percibe riesgo menor \\
\hline & Las personas influyen en la percepción & No influye quien los produzca \\
\hline \multirow{5}{*}{ 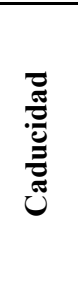 } & Son perecederos & Pueden no ser perecederos \\
\hline & No pueden inspeccionarse & Pueden inspeccionarse \\
\hline & $\begin{array}{l}\text { No tienen una vida, tienen dimensión } \\
\text { temporal }\end{array}$ & $\begin{array}{l}\text { No tienen dimensión temporal, pero si } \\
\text { tienen vida, utilidad durante un tiempo }\end{array}$ \\
\hline & No se pueden inventariar & Se pueden inventariar \\
\hline & No se pueden devolver & Se pueden devolver \\
\hline
\end{tabular}

Fuente: Elaboración propia. 
Algunas de las observaciones más destacadas en la revisión de la literatura, son el uso o adaptación de escalas ampliamente validadas, como es el caso de la escala SERVQUAL (Parasuraman et al. 1985). Así, de ella se han desprendido diferentes estudios realizados en el sector bancario (Rodríguez Parada 1993; Yavas et al. 1997; Bahia y Nantel 2000; Fernández Barcala 2000; Allred y Addams 2000; Saurina 2002; Jayawardhena 2004; Yavas et al. 2004; Arasli et al. 2005; Bath 2005; Karatepe et al. 2005), en donde inicialmente toman las cinco dimensiones de SERVQUAL (elementos tangibles, fiabilidad, capacidad de respuesta, seguridad y empatía), así como sus 22 ítems.

Para autores como Cronin y Taylor (1992) la escala SERVQUAL no es válida para medir la calidad del servicio, ni la satisfacción del consumidor, puesto que el marco conceptual en el que se sustenta, no se basa en el modelo de las actitudes sino en uno de percepcionesexpectativas. Estos autores crean a partir de ésta su escala SERVPERF, en donde tienen en cuenta tan sólo las percepciones de los clientes, pues argumentan que la calidad del servicio basada solo en el resultado es un mejor instrumento para medir la calidad del servicio.

En el sector bancario también se ha usado la escala SERVPERF, realizando las preguntas en las que se tienen únicamente en cuenta las percepciones (García Mestanza 1998; Jabnoun y Al-Tanami 2003; Ting 2004; Sharma y Mehta 2004; Bauer et al. 2005).

También existen estudios en donde se trabaja con las dos escalas (SERVQUAL y SERVPERF), en sus versiones originales y ponderadas, intentando buscar la escala que ofrezca mayor validez (Angur et al. 1999; Chi Cui et al. 2003).

Por otra parte, se han realizado estudios teniendo en cuenta el modelo de la imagen de Grönroos $(1983,1994)$ en donde la calidad del servicio es el resultado de integrar la calidad total en tres tipos de dimensiones (calidad técnica, calidad funcional e imagen corporativa), que condicionan la percepción que un sujeto tiene de un objeto, sea producto o servicio; este modelo se ha usado como única referencia (Aldlaigan y Buttle 2002) o en combinación con la escala SERVQUAL (Lassar et al. 2000).

Y a pesar de las escalas tenidas en cuenta como referencia, otros investigadores de la calidad del servicio en el sector de la banca han creado sus propias escalas (Lewis 1993; Jamal y Nasser 2002; Gounaris et al. 2003; Sureshchandar et al. 2003; Paswan et al. 2004; Al-Hawari et al. 2005). 
Con la ayuda de estos modelos podemos definir la calidad del servicio bancario como la esmerada y correcta entrega del servicio bancario, que busca conseguir la satisfacción de los clientes, y en el que debe trabajar conjuntamente toda la organización. En ella influyen diferentes factores.

En primer factor recoge los aspectos físicos, tiene en cuenta la estructura física, la ubicación conveniente, el equipamiento moderno de los equipos, el ambiente interno y la disposición de los recursos, que provocan un impacto positivo sobre los clientes (Sharma y Mehta 2004: 218).

- H1a: Los aspectos físicos que existen en la prestación del servicio influyen directamente en la calidad del servicio percibida por los clientes de las entidades bancarias de Castilla y León.

El segundo factor recoge los aspectos del desempeño operativo de la prestación del servicio, tales como el tiempo de entrega del servicio o la puntualidad del mismo, actitudes y capacidades de los empleados en la prestación del servicio, la atención personalizada, o la confianza y el conocimiento del personal; también incluye aspectos financieros, como por ejemplo, si los productos y servicios que ofrece la entidad son acordes a las necesidades de los clientes, si el banco ofrece diferentes tipos de préstamos u ofrece una buena rentabilidad frente a otras entidades.

- H1b: Los aspectos de la prestación del servicio de la entidad influyen directamente en la calidad del servicio percibida por los clientes de las entidades bancarias de Castilla y León.

En tercer lugar, se ha elaborado una dimensión que ha venido tomando fuerza en los últimos años, pues en un entorno tan competitivo como el actual, las entidades bancarias deben perseguir el logro de una ventaja competitiva basada en las tecnologías de la información (Larrán y Muriel 2004: 82). Por ello, a medida que avancen las tecnologías, la entidad debe perseguir avanzar en la automatización de los servicios. Se le ha denominado "nuevas tecnologías" e incluye tres tipos de servicios automatizados: i) el servicio de los cajeros automáticos, que si bien es un aspecto que lleva muchos años en la rutina bancaria, y no parece adecuado llamarlo nueva tecnología, la cantidad de servicios que cada vez se pueden recibir en los cajeros hace que se opte por incluirla aquí; ii) banca en Internet (Jawardhena 2004), y iii) banca telefónica (Al-Hawari 
et al. 2005), que ayudan a dar a conocer al banco y a agilizar las transacciones de los clientes, disminuyendo la saturación en las oficinas.

- H1c: Las nuevas tecnologías que ofrece la entidad influyen directamente en la calidad del servicio percibida por los clientes de las entidades bancarias de Castilla y León.

Por último, se trata de verificar si estos aspectos que influyen en la calidad del servicio, se correlacionan entre sí.

- H1d: Los factores determinantes del servicio se correlacionan fuertemente entre sí.

\subsection{Calidad y Satisfacción en el servicio bancario}

El estudio de la satisfacción del cliente, tanto desde un punto de vista conceptual como de medición operativa, contribuyen a avanzar en el conocimiento, pero la falta de coincidencia en la conceptualización de la satisfacción supone para los investigadores cierta dificultad a la hora de seleccionar una definición adecuada, desarrollar medidas válidas y comparar e interpretar los resultados empíricos (Peterson y Wilson 1992; Giese y Cote 2000). Sin embargo, a pesar de la cantidad de definiciones aún persiste la inexistencia de una definición uniforme de la satisfacción, lo que puede ocasionar graves perjuicios en las investigaciones (Gardial et al. 1994; Peterson y Wilson 1992; Yi 1991).

Los términos 'satisfacción' y 'calidad' están interrelacionados; incluso en algunos casos se consideran como sinónimos, hasta el punto que sugieren que los modelos de satisfacción pueden ser denominados de calidad de servicio percibida, ya que lo que se estudia es un servicio y no un bien de consumo.

A pesar de que en ambos casos hablamos de evaluaciones subjetivas por parte de los clientes o usuarios, es importante destacar ciertas diferencias, señalando que las investigaciones realizadas sobre satisfacción se han centrado en las evaluaciones posteriores al consumo o compra, mientras que las investigaciones sobre actitudes han enfatizado la atención en evaluaciones anteriores a la decisión de consumo o compra. Algunas de sus diferencias más notorias las encontramos en el Cuadro 2.

Satisfacción y calidad del servicio son constructos diferentes que han obtenido diversas posiciones respecto a su ordenamiento causal. Teniendo en cuenta que la satisfacción del cliente es un objetivo 
importante para ser alcanzado en las entidades bancarias y, que una manera de conseguirla es hacerlo mediante la calidad de servicio (Goode et al. 1996), consideraremos al igual que en la mayoría de las investigaciones de este tipo, a la calidad de servicio como el antecedente de la satisfacción (Cronin y Taylor 1992; Anderson y Sullivan 1993; Anderson et al. 1994; Taylor y Baker 1994; Leunissen et al. 1996; Lloréns 1996; Bigné et al. 1997; Yavas et al. 1997; Jamal y Nasser 2002; Lassar et al. 2000).

- H2: La calidad del servicio influye positiva y directamente en la satisfacción del cliente de las entidades bancarias de Castilla y León.

Cuadro 2: Calidad vs. Satisfacción

\begin{tabular}{|c|c|c|}
\hline & Calidad & Satisfacción \\
\hline Definición & $\begin{array}{l}\text { Tipo de actitud apoyado en } \\
\text { una valoración general a lo } \\
\text { largo del plazo. }\end{array}$ & $\begin{array}{l}\text { Juicio transitorio de encuentro } \\
\text { específico de servicio. }\end{array}$ \\
\hline Base & $\begin{array}{l}\text { Se basa en las percepciones } \\
\text { ideales o de excelencia }\end{array}$ & $\begin{array}{l}\text { Proceso basado en la disconformidad de } \\
\text { expectativas }\end{array}$ \\
\hline Nivel de análisis & $\begin{array}{l}\text { Concepto global para el bien } \\
\text { o servicio. }\end{array}$ & $\begin{array}{l}\text { Se verifica en cada transacción o } \\
\text { consumo de un bien o servicio. }\end{array}$ \\
\hline Valoración & $\begin{array}{l}\text { Para la valoración no } \\
\text { requieren experiencia antes } \\
\text { del consumo del servicio. }\end{array}$ & $\begin{array}{l}\text { Solo se puede valorar después de una } \\
\text { experiencia de consumo. } \\
\text { El totalmente experimental; sólo } \\
\text { después de una experiencia de consumo } \\
\text { es posible valorarla. }\end{array}$ \\
\hline $\begin{array}{l}\text { Dimensiones que } \\
\text { forman juicios }\end{array}$ & $\begin{array}{l}\text { Se basan en atributos o } \\
\text { indicadores y por eso son mas } \\
\text { especificas. }\end{array}$ & $\begin{array}{l}\text { Pueden resultar de cualquier dimensión, } \\
\text { esté relacionada con la calidad o no. }\end{array}$ \\
\hline $\begin{array}{l}\text { Antecedentes } \\
\text { conceptuales }\end{array}$ & $\begin{array}{l}\text { Posee procesos cognitivos, } \\
\text { aunque comunicaciones } \\
\text { personales e impersonales } \\
\text { juegan un mayor papel. }\end{array}$ & $\begin{array}{l}\text { Es influenciada por un mayor número } \\
\text { de procesos cognitivos y afectivos entre } \\
\text { los que se incluyen la equidad, } \\
\text { atribuciones y emociones. }\end{array}$ \\
\hline Expectativas & $\begin{array}{l}\text { Son vistas como deseos o } \\
\text { necesidades de los clientes. }\end{array}$ & $\begin{array}{l}\text { Son predicciones hechas por los clientes } \\
\text { sobre lo que les gustaría que sucediera } \\
\text { durante un intercambio. }\end{array}$ \\
\hline $\begin{array}{l}\text { Valoraciones que } \\
\text { hace el cliente }\end{array}$ & $\begin{array}{l}\text { Resultan de comparar entre el } \\
\text { servicio deseado y el servicio } \\
\text { percibido. }\end{array}$ & $\begin{array}{l}\text { Resultan de la comparación entre el } \\
\text { servicio previsto y el servicio percibido. }\end{array}$ \\
\hline
\end{tabular}

Fuente: Elaboración propia. 


\subsection{Satisfacción y Lealtad en el servicio bancario}

Dada la nueva orientación del mercado, todas las organizaciones reconocen la importancia del valor añadido del servicio y de las relaciones con los clientes con un objetivo básico: mantener la lealtad de los mismos (Barroso 1995: 196). Pero hay que tener en cuenta que no siempre los efectos de la satisfacción de los clientes se manifiestan en comportamientos positivos (Jacoby y Jaccard 1981) y pueden existir clientes que, aún estando satisfechos con encuentros sucesivos, no tienen ninguna lealtad a la entidad, y cambian fácilmente a la competencia, posiblemente por la ausencia de un sentimiento de pertenencia.

Durante las dos décadas pasadas, se ha dedicado una considerable atención a la satisfacción del cliente como un determinante potencial de la lealtad del cliente (Fornell 1992; Oliver 1999), pero exceptuando unas pocas ocasiones, la satisfacción total del cliente es la clave para asegurar su lealtad y generar una relación rentable de largo plazo (Jones y Sasser 1995: 89).

- H3: La satisfacción del cliente influye positiva y directamente en la lealtad del cliente de las entidades bancarias de Castilla y León.

Podemos ver de manera gráfica las diferentes hipótesis en

la Figura 1.

Figura 1: Hipótesis de la investigación

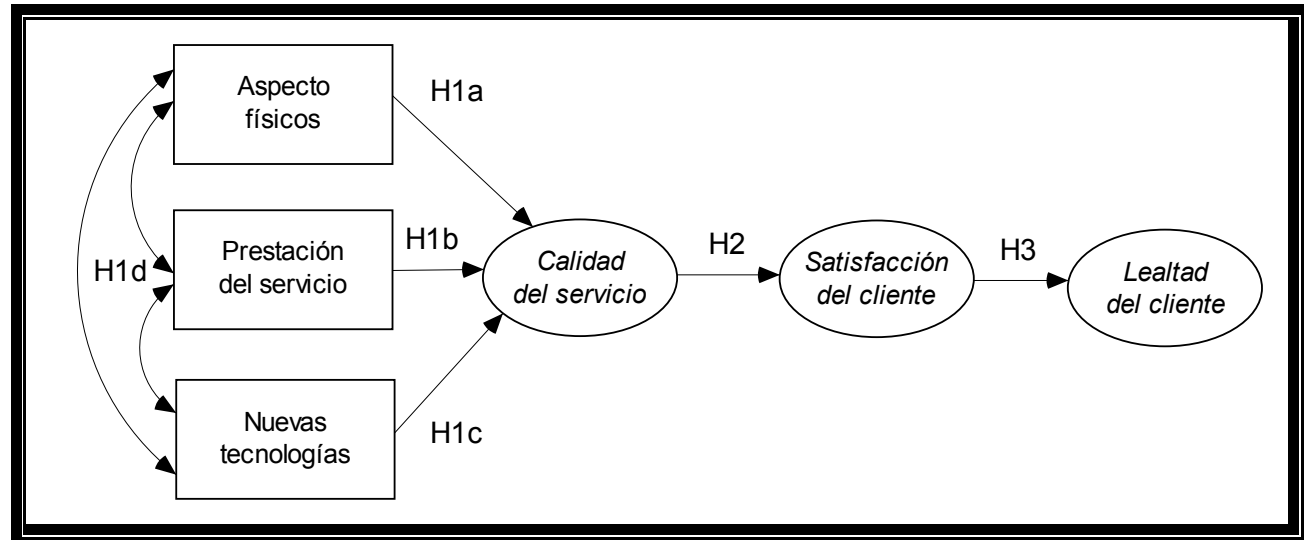

Fuente: Elaboración propia. 


\section{METODOLOGÍA}

A partir de la revisión de la literatura sobre las diferentes escalas para su medición, se desarrolló una escala propia de medición de la calidad del servicio, adaptada específicamente al sector de los servicios bancarios.

Las encuestas personales (ver Anexo) se realizaron proporcionalmente por provincia, teniendo en cuenta el número de habitantes y su proporción de acuerdo a la población total en la región de Castilla y León. En el Cuadro 3 se recoge, a modo de resumen, la ficha técnica de la investigación y en Miguel-Dávila y Flórez-Romero (2007) se presenta un mayor desarrollo de los resultados descriptivos del estudio.

Cuadro 3: Ficha técnica del estudio

\begin{tabular}{|c|c|}
\hline Objeto evaluado & Calidad del servicio prestado por las entidades financieras \\
\hline Sujeto evaluador & $\begin{array}{l}\text { - Clientes con al menos una cuenta con alguna entidad financiera } \\
\text { presente en Castilla y León } \\
\text { - Mayor de } 18 \text { años }\end{array}$ \\
\hline Ámbito geográfico & Comunidad de Castilla y León \\
\hline Tamaño muestral & 400 cuestionarios \\
\hline Diseño muestral & Entrevistas personales \\
\hline Error muestral & $5 \%$ (para el caso más desfavorable y para un nivel de confianza del $95 \%$ ) \\
\hline Trabajo de Campo & Junio y Octubre de 2006 \\
\hline
\end{tabular}

Fuente: Elaboración propia.

Esta investigación ha seguido la metodología para la creación de escalas de medida habituales en las ciencias sociales (Churchill 1979), con una aplicación secuencial, dando comienzo con una idea que va acotándose, y que, una vez delimitada, se procede al establecimiento de los objetivos y preguntas de la investigación.

Nuestra intención en la investigación es la especificación del dominio de los constructos 'calidad del servicio', 'satisfacción' y 'lealtad del cliente'. Para ello, hemos utilizado la recolección de datos para pasar a verificar las hipótesis con base en la medición numérica y el análisis estadístico para establecer patrones de comportamiento (Hernández Sampieri et al. 2003: 6). 
La depuración de la escala de medida es necesaria, debido a que para que una escala sea un instrumento útil en la medida, tanto de la calidad del servicio como de cualquier actitud, ha de cumplir una serie de propiedades sociométricas, tales como la fiabilidad y la validez (Fernández Barcala 2000: 58).

\section{FIABILIDAD Y LA VALIDEZ DE LA ESCALA}

Para el análisis de la fiabilidad, se utilizó el coeficiente alfa de Cronbach, y el análisis de la matriz de correlaciones para la eliminación de los ítems con baja carga. El ítem X11 (horario de atención) y el ítem X26 (tiempo de ahorro con la banca on-line) fueron eliminados, pasando a tener una fiabilidad de 0,919.

La justificación de la baja correlación de estos ítems, puede deberse, en el primer caso (X11) a que al tener todas las entidades prácticamente el mismo horario, no es un aspecto al que los clientes le presten especial atención, y en el segundo (X26) al bajo número de clientes que manifiesta que aún no maneja la página Web, por lo que prefiere acercarse a su sucursal, no pudiendo apreciar el ahorro de tiempo que ésta ofrece.

Por su parte, la validez de una escala nos indica en qué grado estamos midiendo lo que en realidad nos proponemos medir. En otras palabras, permite conocer si dichas escalas son capaces de medir aquello (variables o constructos) para lo que han sido concebidas (Visauta 1998: 298). Hemos hallado para la investigación la validez de contenido y la validez de constructo.

La validez de contenido se aplica para estimar si el procedimiento seguido para la elaboración de la escala de medida ha sido el adecuado (Peter y Churchill 1986: 1). En general se acepta si la escala ha sido desarrollada a partir de las teorías existentes en la literatura sobre dicha materia. En nuestra investigación, al realizarse una exhaustiva y adecuada revisión de la literatura sobre las escalas creadas, podemos asumir que posee validez de contenido.

La validez de constructo trata de reflejar que existe una relación teórica entre la variable objeto de medida y otras variables (Bollen 1989). Es decir, trata de comprobar si el concepto que se desea 
medir es el que realmente está midiendo la escala. Para hallar este tipo de validez se analiza la validez convergente y la validez discriminante.

La validez convergente existe cuando la medida se correlaciona fuertemente y de forma positiva con otras medidas del mismo constructo (Churchill 1979: 70). Ésta viene influida positivamente por la fiabilidad, de forma que a mayor fiabilidad, mayor validez convergente (Peter y Churchill 1986). Para medir la validez convergente, se utilizaron dos procedimientos, el análisis Factorial Exploratorio (AFE) y Análisis Factorial Confirmatorio (AFC).

Cuadro 4: Matriz de componentes rotados

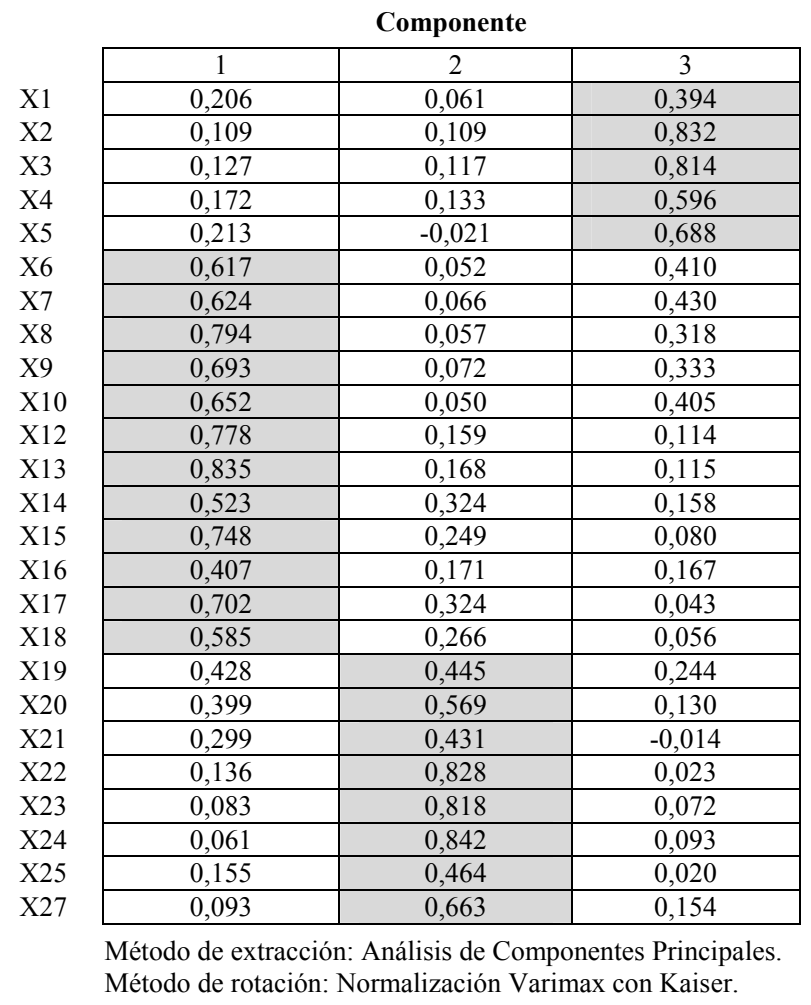

En el AFE, se determinaron los factores que componen cada constructo, realizado por medio de un análisis factorial de componentes principales, con la ayuda del paquete estadístico SPSS 13.0 para Windows. En principio, todas las variables cargaban en el primer factor, por lo que se puede afirmar que la escala es básicamente unidimensional. A continuación, se transformó la matriz factorial en una más fácil de 
interpretar, realizándole una rotación ortogonal con el método varimax, produciéndose una distribución de las variables en tres factores, que en su conjunto representan el $53,08 \%$ de la varianza total de las variables originales (Cuadro 4).

En el primer componente cargan las variables de X6 a X18, las cuales manifiestan aspectos referentes a las operaciones y servicios convencionales que realiza y ofrece la entidad (prestación del servicio). El segundo incluye las variables de X19 a X27, que valoran los aspectos relacionados con los servicios automatizados en la entidad para la prestación del servicio (nuevas tecnologías). Por último, el tercer componente recoge las variables de $\mathrm{X} 1$ a X5, que hacen referencia al aspecto físico de la entidad.

En una investigación similar en entidades bancarias colombianas, se obtuvieron cuatro factores (Valdunciel et al. 2007): aspectos operativos, aspectos físicos, nuevas tecnologías y aspectos humanos.

La constitución de los factores obtenida del AFE se introdujo en un modelo confirmatorio, estimado por medio de ecuaciones estructurales mediante Amos 6.0. Se comprobó que los coeficientes estandarizados de los ítems que componen la escala eran superiores a 0,5 y significativos $(p<0,05)$, lo cual permite aceptar la existencia de la validez convergente.

Por su parte, la validez discriminante indica en qué grado dos medidas desarrolladas para medir constructos similares pero conceptualmente diferentes están relacionadas (Bearden et al. 1993). Mediante un análisis de correlación entre los constructos, se ha medido la validez discriminante, comprobando que en ninguna correlación tenía como valor "1", es decir, que ninguno de los ítems que formaban parte de los diferentes factores aparecía en los otros. Por tanto, con este resultado se entiende que la escala de medición de la calidad posee validez discriminante.

\section{RESULTADOS DEL MODELO DE ECUACIONES ESTRUCTURALES}

La aplicación de los modelos de ecuaciones estructurales ofrece suficiente flexibilidad al investigador, convirtiéndose en una poderosa herramienta de análisis para muchos objetivos de la investigación (Hair et al. 1999). Esta técnica es adecuada para el análisis de sistemas de 
relaciones, validando en primer lugar los componentes del sistema y, en segundo lugar, determinando la relación entre los componentes. Las relaciones causales que explican los MEE son las que se realizan entre un conjunto de variables latentes -no observables- cada una de ellas medida por una o más variables manifiestas -observables- (Diamantopoulos 1994: 105). En este modelo, las variables latentes son los tres aspectos que influyen en la calidad del servicio, mientras que las variables manifiestas son la calidad del servicio, la satisfacción y la lealtad del cliente.

Figura 2: Diagrama de paso del modelo estructural con resultado del modelo causal

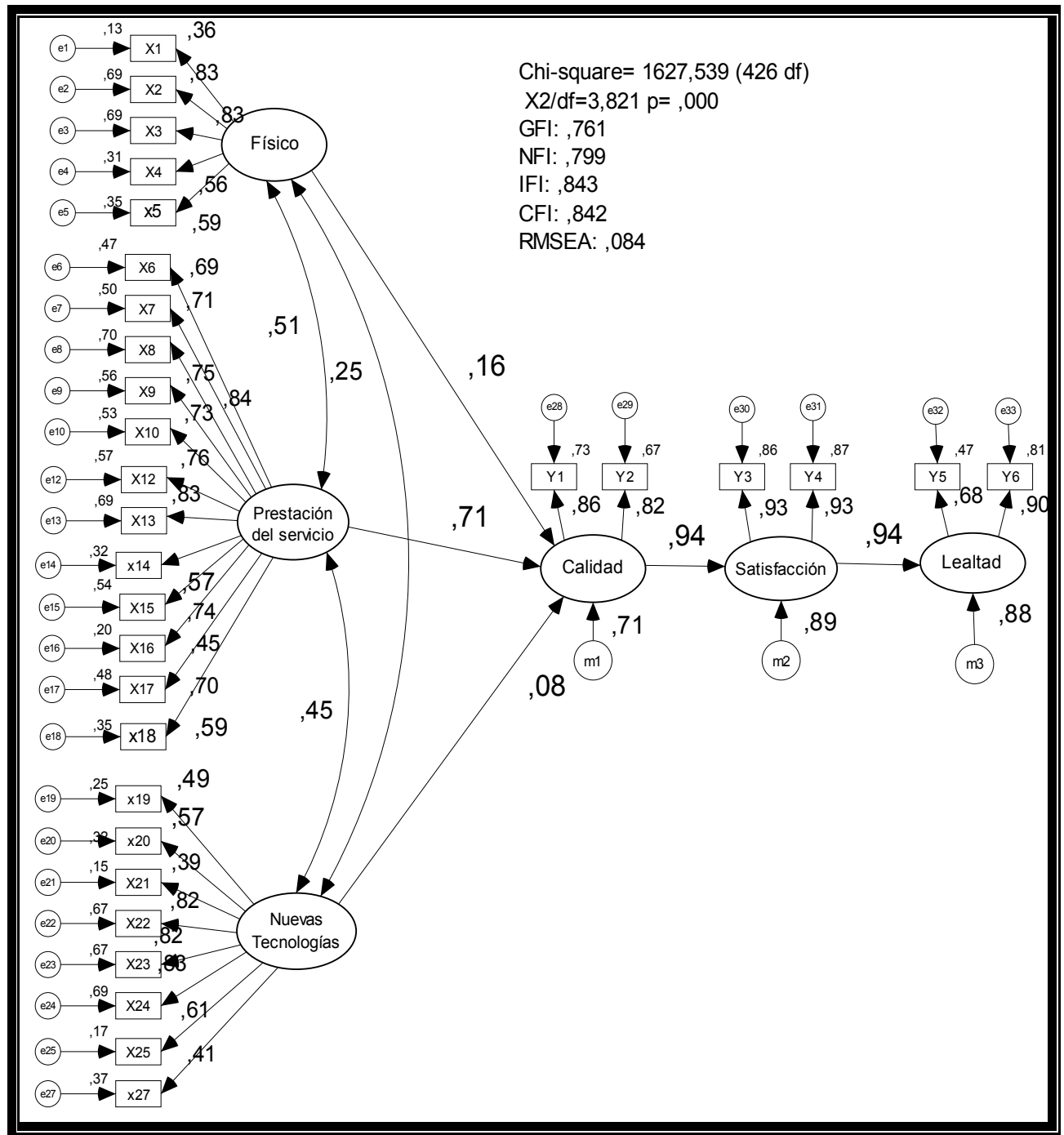

Fuente: Elaboración propia. 
Para determinar este modelo hemos utilizado el programa AMOS 6.0 que permite resolver los modelos de manera gráfica, ecuacional y direccional. Con él, se ha realizado el AFC que se puede observar de manera gráfica en la Figura 2.

Tomando los valores que aparecen en el diagrama de paso, quedan verificadas las hipótesis referentes a las variables exógenas: $\mathrm{H} 1 \mathrm{a}$, $\mathrm{H} 1 \mathrm{~b}$ y H1c, así como la $\mathrm{H} 1 \mathrm{~d}$, ya que los diferentes aspectos que influyen en la calidad del servicio se encuentran intercorrelacionados entre sí. Con respecto a las hipótesis de las variables endógenas $\mathrm{H} 2$ y H3 , se han podido verificar, con valores de 0,94 . Todos los valores tienen un $p$ value de 0,00 , salvo los de la hipótesis $\mathrm{H} 1 \mathrm{c}$ con un $\mathrm{p}<0,05$.

Ahora bien, para validar un modelo conviene realizar diversas reespecificaciones del propio modelo y analizar una serie de índices que son indicativos de su consistencia estadística (Lévy 2000: 194). Por esta razón, a pesar de que las hipótesis se han podido contrastar con este modelo, se opta por realizar una reespecificación del modelo para mejorar los índices de ajuste (los cuales serán buenos cuanto más se acerquen a "1", a excepción del RMSEA que debe ser inferior a 0,05).

Esta fase de reespecificación consiste en intentar mejorar el ajuste obtenido mediante sucesivas modificaciones del modelo, en las cuales se procederá a eliminar aquellas variables que no sean representativas o que conlleven problemas de multicolinealidad o de duplicaciones de la información, así como las relaciones no significativas entre variables latentes.

Para ello, se utilizan los índices de modificación que proporcionan los distintos programas estadísticos. Estos índices sugieren todas aquellas relaciones que conducen a mejorar el modelo. Así, la existencia de saturaciones cruzadas será indicativa de problemas de multicolinealidad, siendo necesario reespecificar el modelo eliminando las variables que presenten estos síntomas. Con ello, se eliminan 13 variables dando como resultado un modelo final más sencillo y mejores índices de bondad del modelo (ver Figura 3).

Los índices del modelo han mejorado considerablemente con respecto al modelo inicial. Con respecto a los valores de la relación de la variable nuevas tecnologías con la calidad, también ha mejorado pasando a ser estadísticamente significativa, aunque es la que menor influencia ejerce en la calidad. Por su parte, la dimensión prestación del servicio es la que más influencia tiene sobre la calidad del servicio. En 
cuanto a las variables endógenas, las relaciones siguen estando por encima del 0,90, pudiéndose verificar las hipótesis en las que la calidad influye en la satisfacción y ésta en la lealtad.

Figura 3: Modelo reespecificado

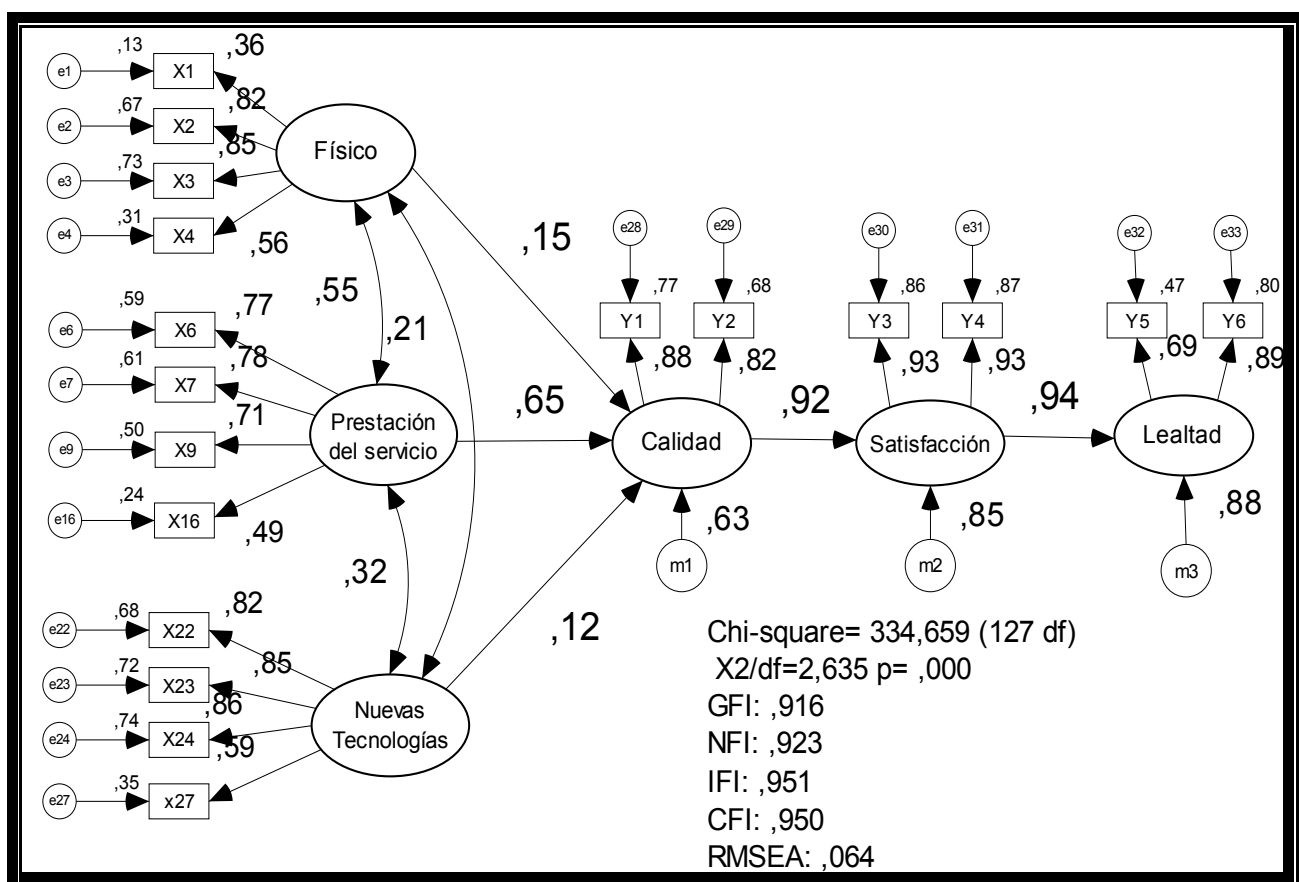

Fuente: Elaboración propia.

\section{CONCLUSIONES}

Partiendo de las escalas existentes, se han creado las dimensiones que se adaptan de manera más precisa al entorno bancario, donde el aspecto prestación del servicio se relaciona con el desempeño de la prestación del servicio, el aspecto físico tiene que ver con la apariencia de las instalaciones y las nuevas tecnologías incluyen los cajeros automáticos, así como los medios virtuales como Internet y la banca telefónica.

El instrumento de medida ha sido elaborado siguiendo la metodología SERVPERF (Cronin y Taylor 1992) en donde sólo se tienen en cuenta las percepciones que tienen los clientes sobre el servicio que reciben. Para comprobar que las dimensiones establecidas se correspondían 
con lo previamente establecido se utilizó un análisis factorial de componentes principales, determinando que todos los ítems se ajustaban según lo estipulado, a excepción de los ítems X11 (horario de atención al público) y X26 (tiempo ahorrado en la banca on-line), los cuales no resultaron significativos.

Mediante la modelización de ecuaciones estructurales con el programa AMOS, se realizó la verificación de las hipótesis, donde podemos señalar que el aspecto prestación del servicio tiene el valor más alto y que más influye en la calidad del servicio $(\mathrm{H} 1 \mathrm{~b})$. Esto responde a la necesidad del servicio y a que los clientes suelen concebir como calidad del servicio aquello relacionado con el desempeño en las transacciones bancarias, la gama amplia de productos y servicios, la exactitud de las explicaciones, la rentabilidad, etc., restando un poco de importancia al aspecto físico de la entidad, a la parte visual y a la apariencia de los empleados $(\mathrm{H} 1 \mathrm{a})$, pues lo que realmente les importa son otros aspectos más cruciales para ellos.

Es decir, para los clientes de entidades bancarias de la Comunidad de Castilla y León, un servicio bien prestado, con una atención personalizada y confianza y conocimiento que presente el personal, junto a la reputación y la solvencia financiera del banco, es sinónimo de calidad.

También podemos observar cómo el avance de las nuevas tecnologías ejerce influencia, aunque pequeña, en la percepción de calidad, lo que se justifica por el impacto obtenido por los medios virtuales en los últimos tiempos que facilitan la relación de las entidades con los clientes, ya que facilitan las transacciones, como la consulta de saldo por teléfono o transferencias por medio de la banca on-line $(\mathrm{H} 1 \mathrm{c})$. Esta menor influencia se justifica porque muchos de los encuestados manifiestan desconfianza o desconocimiento de estos medios (en menor caso, los cajeros automáticos), por lo que a pesar del impacto obtenido por los medios virtuales en los últimos tiempos, muchos prefieren seguir utilizando las sucursales tradicionales, lejos de obtener las ventajas de los medios virtuales, como la más importante: el ahorro de tiempo.

Los valores significativos de las intercorrelaciones de los aspectos que influyen en la calidad del servicio sugieren que estos indicadores son correctos ( $\mathrm{H} 1 \mathrm{~d})$, siendo los aspectos físicos y los de la prestación del servicio los que presentan una mayor correlación. 
En cuanto a la calidad y la satisfacción, el alto valor conseguido, indica que en gran medida los clientes que perciben calidad del servicio, quedarán satisfechos con la entidad. Por tanto, la calidad del servicio es un antecedente de la satisfacción del cliente $(\mathrm{H} 2)$. Aunque la evidencia empírica no lo respalde totalmente, podemos concluir que los clientes que están satisfechos con la entidad, es muy probable que le sean leales, continuando su relación con la misma $(\mathrm{H} 3)$.

En resumen, la investigación presentada ha sido el resultado de una revisión de la literatura en materia de calidad del servicio aplicada al sector específico bancario en Castilla y León. Las limitaciones teóricas y metodológicas que se pueden señalar suponen oportunidades de estudio que pueden ir mejorando la labor investigadora.

El marco geográfico de la investigación (Comunidad de Castilla y León) podría ser utilizado en un ámbito más extenso. Lo mismo ocurre en el caso del sector utilizado (bancario), puesto que podrían realizarse modificaciones y adaptaciones para ser aplicado en otros tipos de servicios prestados por sectores similares (seguros, mutuas, etc.), los cuales pueden que no aporten los mismos resultados.

Por último, la mayoría de las variables observables se basan en percepciones de los clientes encuestados, lo que puede generar ciertos sesgos, puesto que alguna de las reacciones positivas o negativas pueden ser resultado de la acumulación de varias situaciones satisfactorias/ insatisfactorias y no estar ligadas a la experiencia específica que se le está solicitando valorar.

\section{BIBLIOGRAFÍA}

AldLAIGAN, A. \& F. ButTLE (2002) "SYSTRA-SQ: A New Measure of Bank Service Quality", International Journal of Service Industry Management, Vol. 13, 4, pp. 362-381.

Al-HaWARI, M.; N. HARTLEY \& T. WARD (2005) "Measuring Banks' Automated Service Quality: A Confirmatory Factor Analysis Approach", Marketing Bulletin, 16, Articule 1, pp. 1-19.

Allred A.T. \& H.L. Addams (2000) "Service Quality At Banks And Credit Unions: What Do Their Customer Say?", Managing Service Quality, Vol. 10, 1, pp. 52-60. 
ANDERSON, E.W. \& M. Sullivan (1993) "The Antecedents and Consequences of Customer Satisfaction for Firms", Marketing Science, Vol. 12, 2, pp. 125-143.

-, C. Fornell y D.R. Lehmann (1994) "Customer Satisfaction, Market Share, and Profitability: Findings from Sweden", Journal of Marketing, Vol. 58, 3, pp. 53-66.

ANGUR, M.; R. NATARAAJAN y J. JAHERA (1999) "Service Quality in the Banking Industry: An Assessment in a Developing Economy", International Journal of Bank Marketing, Vol. 17, 3, pp. 116-123.

ARASLI, H.; S.T. KatiRCIOGLU \& S. MehtAP-Smadi (2005) "A Comparison of Service Quality in the Banking Industry. Some Evidence from Turkishand Greek-speaking Areas in Cyprus", International Journal of Bank Marketing, Vol. 23, 7, pp. 508-526.

BAHIA, K. y J. NANTEl (2000) "A Reliable and Valid Measurement Scale for the Perceived Service Quality of Banks", International Journal of Bank Marketing, Vol. 18, 2, pp. 84-81.

BARROSO, C. (1995) El marketing bancario. Un enfoque estratégico. Madrid: Esic.

BATH, M.A. (2005) "Corelates of Service Quality in Banks: An Empirical Investigation", Journal of Services Research, Vol. 5, 1, pp. 77-99.

BAUER, H.H.; M. HAMmerschmidT \& T. FAlK (2005) "Measuring the Quality of e-Banking Portals", International Journal of Bank Marketing, Vol. 23, 2, pp. 153-175.

Bearden, W.O.; R.G. Netemeyer \& M.F. Mobley (1993) Handbook of Marketing Scales. Multi-item Measures for Marketing and Consumer Behaviour Research. Newbury Park: Sage Publications.

Bigné, J.E.; M.A. MOliner, T.M. VAllet y J. SÁnChez (1997) "Un estudio comparativo de los instrumentos de medición de la calidad de los servicios públicos", Revista Española de Investigación de Marketing ESIC, (Septiembre), pp. 33-53.

BOLLEN, K.A. (1989) Structural Equations with Latent Variables. New York: John Wiley \& Sons.

CHI CUI, C.; B.R. LeWIS \& W. PARK (2003) "Service Quality Measurement in the Banking Sector in South Korea", International Journal of Bank Marketing, Vol. 21, 4, pp. 191-201.

CHURCHILL, G.A., Jr. (1979) "A Paradigm for Developing Better Measures of Marketing Constructs", Journal of Marketing Research, Vol. XVI (February), pp. 64-73. 
CRONIN, J.J., Jr. y S.A. TAYLOR (1992) "Measuring Service Quality: A Reexamination and Extension", Journal of Marketing, Vol. 56 (Julio), pp. $55-68$.

DIAMANTOPOULOS, A. (1994) "Modelling with LISREL: A Guide for the Uninitiated", Journal of Marketing Management, Vol. 10, 1-3, pp. 105136.

FERNÁNDEZ BARCALA, M. (2000) "Validación de SERVQUAL como instrumento de medida de la calidad de servicio bancario", Revista Europea de Dirección y Economía de la Empresa, Vol. 9, 1, pp. 57-70.

FORNELL, C. (1992) "A National Customer Satisfaction Barometer: The Swedish Experience", Journal of Marketing, Vol. 56 (January), pp. 6-21.

García MestanZA, J. (1998) Calidad del servicio financiero como estrategia de las instituciones bancarias. Málaga: Unicaja.

GaRdiaL, S.F.; D.S. Clemons, R.B. WOOdRufF, D.W. SCHUMAN \& M.J. BuRnS (1994) "Comparing Consumer's Recall of Prepurchase and Postpurchase Product Evaluation Experiences", Journal of Consumer Research, Vol. 20 (March), pp. 548-560.

GAYATHRI, H.; M.C. VinAYA \& K. LAKSHMisha (2005) "A Pilot Study on the Service Quality of Insurance Companies", Journal of Services Research, Vol. 5, 2 (October), pp. 123-138.

GIESE, J.L. \& J.A. COTE (2000) "Defining Consumer Satisfaction", Academy of Marketing Science Review, Vol. 1, pp. 1-34.

GONZÁlEZ APONCIO, Z. (2001) El reto de la calidad del servicio financiero en la Comunidad Autónoma de Canaria. Santa Cruz de Tenerife: Fyde Cajacanarias.

GOUNARIS, S.P.; V. STATHAKOPOULOS \& A.D. ATHANASSOPOULOS (2003) "Antecedents to Perceived Service Quality: An Exploratory Study in the Banking Industry", International Journal of Bank Marketing, Vol. 21, 4, pp. 168-191.

GRÖNROOS, C. (1983) Strategic Management and Marketing in the Service Sector. Cambridge. MA: Marketing Science Institute.

- (1994) Marketing y gestión de servicios. Madrid: Díaz de Santos.

GummesSON, E. \& C. GRÖNROOS (1988) "Quality of Services. Lessons from the Product Sector". C. SURPRENANT (ed.) Add Value to Your Service. Chicago, IL.: American Marketing Association.

Hair, J.F.; R.E. ANDERson, R.L. TAtham \& W.C. BLACK (1999) Análisis Multivariante. Madrid: Prentice Hall. 
Hernández Sampieri, R.; C. Fernández Collado y P. Baptista lucio (2003) Metodología de la investigación, $3^{a}$ ed.. México: McGraw-Hill.

Jabnoun, N. \& H. Al-Tamimi (2003) "Measuring Perceived Service Quality at UAE Commercial Banks", International Journal of Quality \& Reliability Management, Vol. 20, 4, pp. 458- 472.

JACOBY, J. \& J.J. JACCARD (1981) "The Sources, Meaning and Validity of Consumer Complaining Behaviour: A Psychological Analysis", Journal of Retailing, Vol. 57, pp. 4-24.

JAMAL, A. \& K. NASSER (2002) "Customer Satisfaction and Retail Banking: An Assessment of Some of the Key Antecedents of Customer Satisfaction in Retail Banking", International Journal of Bank Marketing, Vol. 20, 4, pp. 146-160.

JONES, T.O. \& W.E. SASSER (1995) "Why Satisfied Customers Defect", Harvard Business Review, Vol. 73, 6, pp. 88-99.

KARATEPE, O.M.; U. YAVAS \& E. BABAKUS (2005) "Measuring Service Quality of Banks: Scale Development and Validation", Journal of Retailing and Consumer Services, Vol. 12, pp. 373-383.

LARRÁN, M. y M.J. MURIEL (2004) "El impacto de las nuevas tecnologías de la información en el negocio bancario español", Revista de Empresa, Vol. 9 (Julio-Septiembre), pp. 78-86.

LASSAR, W.M.; C. MANOLIS \& R. WINDSOR (2000) "Service Quality Perspectives and Satisfaction in Private Banking", Journal of Services Marketing, Vol. 14, 3, pp. 244-271.

LeUnissen, P.; O. PReVo \& H. ROEST (1996) "The Mediating Role of Psychosocial Benefits in the Satisfaction Formation Process". J. BERACS, A. BAUER \& J. SIMON (eds.) Proceedings of the 25th Annual Conference of the European Marketing Academy, Marketing for an Expanding Europe. Budapest: Budapest University of Economic Sciences, pp. 1869-1878.

LÉVY, J.P. (2000) Modelización y programación estructural con AMOS. Madrid: Instituto Superior de Técnicas y Prácticas Bancarias. CD-Rom.

LEWIS, B.R. (1993) "Service Quality: Recent Developments in Financial Services", International Journal of Bank Marketing, Vol. 11, 6, pp. 19-25.

LEWIS, R.C. y D.M. KLEIN (1987) "The Measurements of Gaps in Service Quality". CZEPIEL et al. (eds.) The Service Challenge: Integrating for Competitive Advantage. Chicago, IL.: American Marketing Association.

LLORÉNS, F.J. (1996) Medición de la calidad de servicio: una aproximación a diferentes alternativas. Granada: Universidad de Granada. 
MIGUEL-DÁVILA, J.A. y M. FLóREZ-ROMERO (2007) "¿Ofrecen calidad las entidades bancarias de Castilla y León?", Boletín Económico de Castilla y León, № 11, pp. 125-132.

OLIVER, R.L. (1999) "Whence Consumer Loyalty?", Journal of Marketing, Vol. 63 (Special Issue), pp. 33-44.

Parasuraman, A.; V.A. Zeithaml \& L.L. Berry (1985) "A Conceptual Model of Service Quality and Its Implications for Future Research", Journal of Marketing, Vol. 49 (Autumn), pp. 41-50.

Paswan, A.K.; N. SPEARS, R. HASTY \& G. GANESH (2004) "Search Quality in the Financial Services Financial a Contingency Perspective", Journal of Services Marketing, Vol. 18, 5, pp. 324-339.

Peter, J.P. \& G.A. ChURChILL (1986) "Relationship Among Research Design Choices and Psychometric Properties of Rating Scales: A Meta-Analysis", Journal of Marketing Research, Vol. XXIII (February), pp. 1-10.

Peterson, R.A. \& W.R. WILSON (1992) "Measuring Customer Satisfaction: Fact and Artifact", Journal of the Academy of Marketing Science, Vol. 58 (January), pp. 111-124.

RodRíGUez PARADA, S. (1993) Calidad de servicio. Exigencia actual para entidades financieras competitivas. Madrid: Club gestión de calidad.

SAURINA, C. (2002) "Medida de la calidad: Adaptación de la escala SERQUAL al Ámbito Financiero", Revista Española de Financiación y Contabilidad, Vol. XXXI, 113 (Julio), pp. 803-831.

Sharma, A. \& V. Mehta (2004) "Service Quality Perceptions in Financial Services - A Case Study of Banking Services", Journal of Services Research, Vol. 4, 2, pp 205-223.

SHIH, Y.-Y. \& K. FANG (2006) "Effects of Network Quality Attributes on Customer Adoption Intentions of Internet Banking", Total Quality Management, Vol. 17 (January), No. 1, pp. 61-77.

SuRESHCHANDAR, G.S.; C. RAJENDRAN \& R.N. ANANTHARAMAN (2003) "Customer Perceptions of Service Quality in the Banking Sector of a Developing Economy: A Critical Analysis", International Journal of Bank Marketing, Vol. 21, 5, pp 233-243.

TAYLOR, S.A. \& T.L. BAKER (1994) "An Assessment of the Relationship Between Service Quality and Customer Satisfaction in the Formation of Consumer's Purchase Intentions", Journal of Retailing, Vol. 70, 2, pp. 163-178.

TING, D.H. (2004) "Service Quality and Satisfaction Perceptions: Curvilinear and Interaction Effect", The International Journal of Bank Marketing, Vol. 22, 6, pp. 407-420. 
ValdunCiel, L.; M. FlóReZ-Romero \& J.A. Miguel-DÁviLA (2007) "Análisis de la Calidad del Servicio que prestan las entidades bancarias y su repercusión en la satisfacción del cliente y la lealtad hacia la entidad", Revista Asturiana de Economía, №38, pp. 79-107.

VISAUTA, B. (1989) Técnicas de investigación social. Barcelona: Promociones y Publicaciones Universitarias.

YAVAS, U.; M. BENKENSTEIN \& U. STUHLDREIER (2004) "Relationships between Service Quality and Behavioural Outcomes: A Study of Private Bank Customers in Germany", International Journal of Bank Marketing, Vol. 22, 2, pp. 144-157.

-, Z. BILGIN \& D. SHEMWELL (1997) "Service Quality in the Banking Sector in an Emerging Economy: A Consumer Survey", International Journal of Bank Marketing, Vol. 15, 6, pp. 217-223.

YI, Y. (1991) "A Critical Review of Consumer Satisfaction". V.A. ZEITHAML (ed.) Review of Marketing 1900. Chicago, IL.: American Marketing Association, pp. 68-123.

Zeithaml, V.A.; L.L. Berry \& A. Parasuraman (1988) "Communication and Control Processes in the Delivery of Service Quality", Journal of Marketing, Vol. 52 (April), pp. 35-48. 


\section{ANEXO. Codificación y descripción de las variables que conforman el instrumento de medida}

\begin{tabular}{|c|c|}
\hline $\mathrm{X} 1$ & La situación conveniente de localización del banco \\
\hline $\mathrm{X} 2$ & El aspecto atractivo y limpio del banco \\
\hline $\mathrm{X} 3$ & El equipamiento tecnológico avanzado que posee el banco \\
\hline $\mathrm{X} 4$ & $\begin{array}{l}\text { El banco tiene la vigilancia adecuada (videocámaras, agentes de seguridad, } \\
\text { etc.). }\end{array}$ \\
\hline $\mathrm{X} 5$ & La apariencia aseada y elegante de los empleados del banco \\
\hline $\mathrm{X} 6$ & La confianza transmitida por el personal debido a su honestidad y honradez \\
\hline $\mathrm{X} 7$ & $\begin{array}{l}\text { El conocimiento y habilidad que posee el personal, necesaria para la prestación } \\
\text { del servicio }\end{array}$ \\
\hline $\mathrm{X} 8$ & La buena voluntad de personal de la sucursal para ayudar \\
\hline $\mathrm{X} 9$ & La atención personalizada que prestan los empleados \\
\hline $\mathrm{X} 10$ & La amabilidad y cortesía de los empleados \\
\hline $\mathrm{X} 11$ & El horario conveniente de atención al público del banco \\
\hline $\mathrm{X} 12$ & $\begin{array}{l}\text { La gama amplia de productos y servicios bancarios del banco, acorde con las } \\
\text { ultimas innovaciones }\end{array}$ \\
\hline $\mathrm{X} 13$ & El esfuerzo por mantener la ausencia de errores en el desarrollo del servicio \\
\hline $\mathrm{X} 14$ & La corta espera en la entrega del servicio \\
\hline $\mathrm{X} 15$ & y claridad en las explicaciones o informaciones dadas \\
\hline $\mathrm{X} 16$ & ra y buena reputación que posee el banco \\
\hline $\mathrm{X} 17$ & El ofrecimiento de diferentes tipos de préstamos ajustados \\
\hline $\mathrm{X} 18$ & $\begin{array}{l}\text { - Las bajas tasas interés de préstamo ofrecidas con respecto a otros bancos } \\
\text { - Las altas tasas interés de depósito ofrecidas con respecto a otros bancos }\end{array}$ \\
\hline $\mathrm{X} 19$ & $\begin{array}{l}\text { La facilidad y el conocimiento brindados por parte del banco para utilizar los } \\
\text { servicios automáticos }\end{array}$ \\
\hline $\mathrm{X} 20$ & La ubicación de los cajeros automáticos en lugares seguros y convenientes \\
\hline $\mathrm{X} 21$ & La facilidad para utilizar los cajeros automáticos \\
\hline $\mathrm{X} 22$ & La disponibilidad de la información en la banca telefónica \\
\hline $\mathrm{X} 23$ & El corto tiempo de espera en la banca telefónica \\
\hline $\mathrm{X} 24$ & La seguridad en banca telefónica \\
\hline $\mathrm{X} 25$ & La facilidad para navegar dentro en el sitio Web de la banca on-line \\
\hline $\mathrm{X} 26$ & El tiempo que se ahorra con la banca on-line con respecto a las oficinas \\
\hline $\mathrm{X} 27$ & La seguridad en la banca on-line \\
\hline $\mathrm{X} 28$ & La calidad en general percibida del ban \\
\hline $\mathrm{X} 29$ & El banco da el servicio esperado \\
\hline $\mathrm{X} 30$ & El banco satisface sus necesidades \\
\hline $\mathrm{X} 31$ & Su banco le da el servicio que espera \\
\hline $\mathrm{X} 32$ & Considera a su banco como primera opc \\
\hline $\mathrm{X} 33$ & Recomendará su banco a cualquiera que busca su consejo \\
\hline
\end{tabular}

